\title{
Numerical analysis of plasmonic metasurfaces for fluorescence enhancement
}

\author{
Roxana Tomescu, Florin Nita, Stefan Caramizoiu, Veronica Anastasoaie, Dana Cristea
}

National Institute for Research and Development in Microtechnology - IMT Bucharest, 126A, Erou Iancu Nicolae Street, 077190, Voluntari, Ilfov, ROMANIA

\begin{abstract}
The paper presents an extensive numerical analysis performed by three-dimensional (3D) simulations using the finite difference in time and space (FDTD) method to identify the optimal geometry, size and configuration of the nano-antennas that constitute a plasmonic metasurface. The aim was to achieve the highest resonance at various wavelengths (NIR-VIS), for local enhancement of the excitation field and collection efficiency of emitted photons. We investigated ten different types of metals, two shapes (disks and U-shape resonators) and various geometrical parameters for the nanoresonators composing the metasurface. The best results for Rhodamine $6 \mathrm{G}$ excitation and emission were obtained using silver resonators with $105 \mathrm{~nm}$ diameter of the cylinder elements in a rectangular array with a $110 \mathrm{~nm}$ period, and with $110 \mathrm{~nm}$ long $\mathrm{U}$-shape placed at a period of $40 \mathrm{~nm}$.
\end{abstract}

\section{Introduction}

Metasurfaces are arrays of sub-wavelength plasmonic structures that can concentrate the electromagnetic fields in small volumes, well below the diffraction limit, through the excitation of localized surface plasmons [1]. In the last years it was demonstrated that the metasurfaces have the potential to control both the light propagation and emission at the nanoscale, showing thus a great potential for tailoring the excitation and emission processes of fluorescent molecules for quantum emitters and biosensing application [2-4].

The paper presents an extensive numerical analysis performed by three-dimensional (3D) simulations using the finite difference in time and space (FDTD) method to identify the optimal geometry, size and configuration of the nano-structures that constitute a plasmonic metasurface, focusing on achieving the highest resonance at various wavelengths (NIR-VIS), for local enhancement of the excitation field and collection efficiency of emitted photons. The final goal is to attain a considerable enhancement of fluorescent emission of a variety of fluorophores from the visible (VIS) to the near infrared (NIR) spectral domains, based on metasurfaces and explore its applicability in biosensing using metasurface platforms.

\section{Model}

To achieve the purpose of this investigation, we analysed two different geometries for the nanostructures that compose a plasmonic metasurface.

The first proposed structure is composed of metallic nanodisks in a rectangular array patterned on a fused-silica substrate. The second analysed geometry is an array of Ushaped structures. We investigated the influence of the nano-resonator dimensions and material on the local enhancement of the excitation field and collection efficiency of emitted photons.

The geometrical parameters analysis and material studies of the nano-structures were performed applying 3D - FDTD method using the OptiFDTD commercial software developed by Opti Wave Canada. We employed periodic boundary conditions (PBC) on the planes parallel to the propagation direction of radiation. The incident radiation is a Gaussian pulse centred at $532 \mathrm{~nm}$ wavelength (excitation wavelength for Rhodamine 6G) and $552 \mathrm{~nm}$ (emission wavelength) respectively. The refractive index of the metals is modelled by a standard Lorentz-Drude formula. More details about the conditions we use to model and simulate a plasmonic metasurface structure are given in [5].

The elementary cell of the first structure under investigation, together with its geometric characteristics is presented in Figure 1 where $\mathrm{L}_{\mathrm{x}}{ }^{0}$ and $\mathrm{L}_{\mathrm{y}}{ }^{0}$ are the transverse dimensions, $\mathrm{H}_{\mathrm{s}}$ is the thickness of the substrate, $h$ and $\phi$ are the height and the diameter of the nano-cylinder, respectively.

We studied the influence of ten different metals and varied the diameters of the resonators from $50 \mathrm{~nm}$ up to $105 \mathrm{~nm}$ with a $5 \mathrm{~nm}$ step. The metal disks are patterned in a rectangular lattice with a constant of $110 \mathrm{~nm}$. The height of the nanostructures is set at $100 \mathrm{~nm}$.

\footnotetext{
* Corresponding author: stefan.caramizoiu@imt.ro
} 


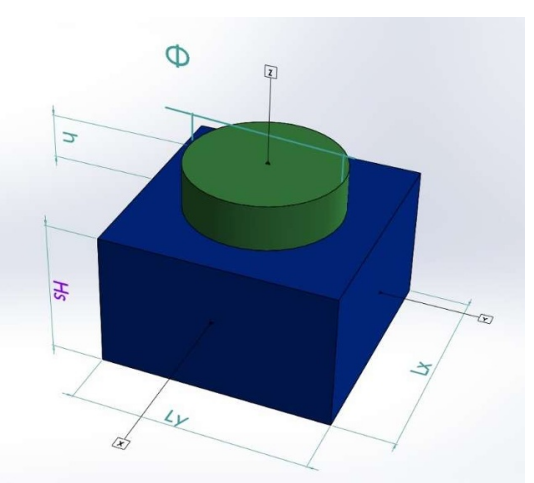

Fig 1. The elementary cell through which can be obtained a metasurfaces of variable dimensions by translation in the two

directions $x$ and $y$. The propagation direction of the electromagnetic radiation is the $z$ direction.

In our second numerical analysis we propose a metasurface composed of an array of U-shaped nanostructures. The materials and simulation conditions employed here are the same as in the first case. The elementary cell of this structure is presented in Figure 2.

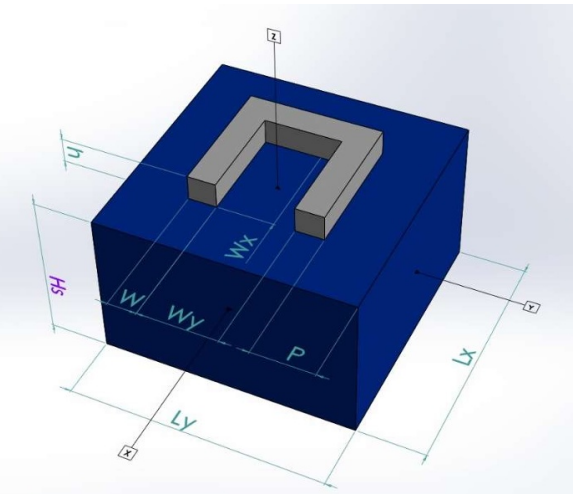

Fig 2. The elementary cell through which can be obtained a metasurfaces with U-shape nanostructures of variable dimensions by translation in the two directions $x$ and $y$. The propagation direction of the electromagnetic radiation is the $z$ direction.

For this type of structures, the local enhancement of the excitation field was studied by varying the dimensions of the structures, or the array period. The geometries employed in three promising sets of 3D simulations are presented in table 1 - where Ly, Lx and $\mathrm{Hs}$ are the geometrical parameters of the substrate, $\mathrm{H}$ is the height of the metallic structure, $\mathrm{P}$ is the distance between the resonator and the boundary of the simulation domain, $\mathrm{W}$ (thickness), Wx (arm length) and Wy (base length) are the parameter of the U-shape structure illustrated in Figure 2.

Table 1. Geometrical parameter used in 3 sets of 3D numerical investigations

\begin{tabular}{|c|c|c|c|c|c|c|c|c|}
\hline $\begin{array}{l}\text { No. } \\
\text { Set }\end{array}$ & $\begin{array}{c}\text { Ly } \\
{[\mathrm{nm}]}\end{array}$ & $\begin{array}{c}\mathrm{Lx} \\
{[\mathrm{nm}]}\end{array}$ & $\begin{array}{c}\mathrm{Hs} \\
{[\mathrm{nm}]}\end{array}$ & $\begin{array}{c}\mathrm{H} \\
{[\mathrm{nm}]}\end{array}$ & $\begin{array}{c}\mathrm{P} \\
{[\mathrm{nm}]}\end{array}$ & $\begin{array}{c}\mathrm{W} \\
{[\mathrm{nm}]}\end{array}$ & $\begin{array}{c}\mathrm{Wx} \\
{[\mathrm{nm}]}\end{array}$ & $\begin{array}{c}\mathrm{Wy} \\
{[\mathrm{nm}]}\end{array}$ \\
\hline 1 & 200 & 200 & 600 & 20 & 50 & 20 & 80 & 60 \\
\hline 2 & 200 & 200 & 600 & 20 & 20 & 20 & 140 & 120 \\
\hline 3 & 200 & 200 & 600 & 20 & 20 & 10 & 110 & 90 \\
\hline
\end{tabular}

\section{Results and discussions}

To identify the best possible result, we analysed the electromagnetic radiation intensity on three different planes: a) interface air/resonators; b) middle of resonator; c) interface resonators/substrate. Examples of results obtained for the excitation wavelength of $532 \mathrm{~nm}$ are given in Figures 3-7.

In Figure 3 one can observe the electromagnetic field intensity function of $\phi$, for the nano-disks metasurface, for all investigated metals. The increase of the field intensity for silver is over 6 times and about 5 times for aluminium at the interface air/resonator for a metasurface with $105 \mathrm{~nm}$ diameter disks.

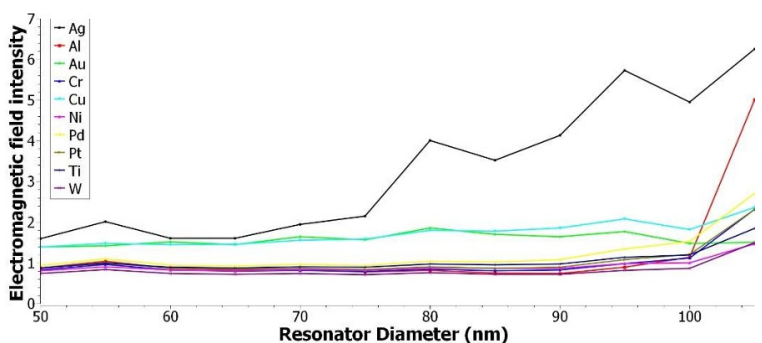

a)

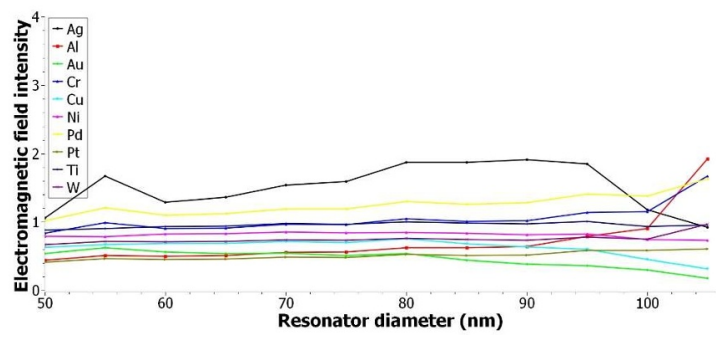

b)

Fig. 3. Electromagnetic field intensity: a) interface medium/resonators; b) interface resonators/substrate.

From the results presented in Figure 3 one can conclude that the appropriate metal for localized fluorescent enhancement is silver which offers optimum increase values for the field intensity for the desired application. In Figure 4 is presented the intensity obtain by modelling a silver metasurface on the three analysed planes.

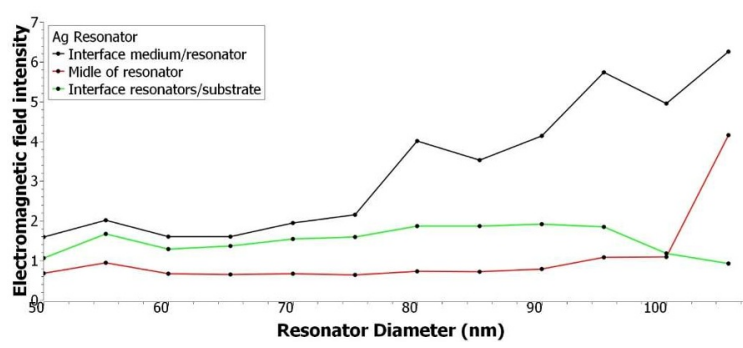

Fig. 4. Electromagnetic field intensity for silver nano-disks metasurface.

Figure 5 shows the electromagnetic field at the two planes for metasurfaces with a 2D squared array of silver nano-disk resonators $105 \mathrm{~nm}$-diameter and $100 \mathrm{~nm}$ - high. 


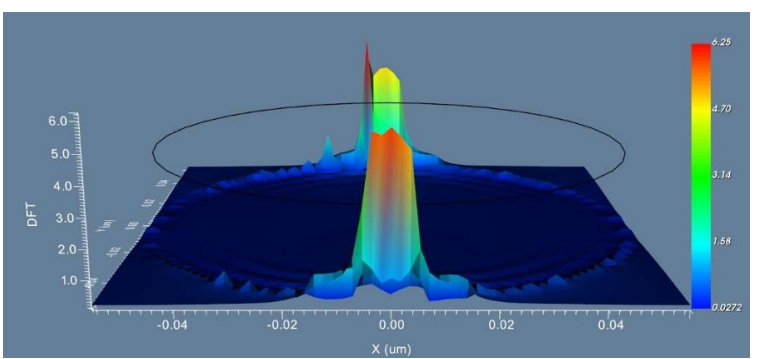

a)

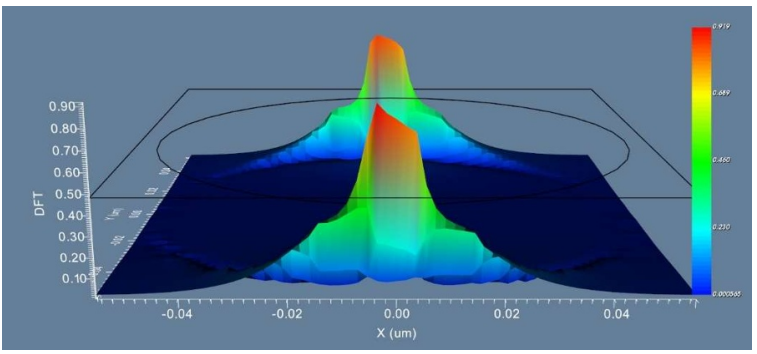

b)

Fig. 5. Electromagnetic field intensity for silver nano-disks metasurface: a) interface medium/resonators; b) on the substrate around the resonator $-105 \mathrm{~nm}$-diameter and $100 \mathrm{~nm}$ high.

One can see from Figures 4 and 5 an important field enhancement both at the interface medium/resonator and on the substrate around the resonator. These results offer the possibility of employing the designed structures in the development of fluorescent based bio-sensing devices.

Following the steps utilised in the first part of our investigation, we performed $3 \mathrm{D}$ simulations of a metasurface structure with U-shape nano-elements as meta-atoms.

The results obtain during this study are presented in figures 6-7. In Figure 6 are given the best electromagnetic field intensities obtain during the investigation performed on the ten selected metals. The graphs represent the results attained performing the third set of simulation for silver and aluminium. Once again, the best choices concerning the materials for the meta-atoms are silver (Figure 6a) and aluminium (Figure 6b). The electromagnetic field localized enchantment goes to values as high as 4.47 on the substrate around a silver U-shape resonator, and around 4 at the medium/resonators for aluminium metaatoms.

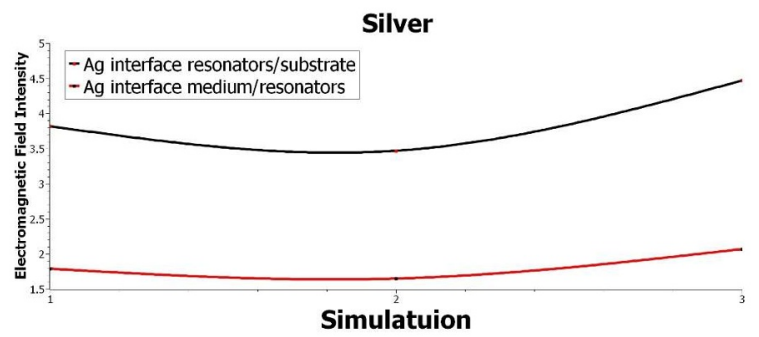

a)

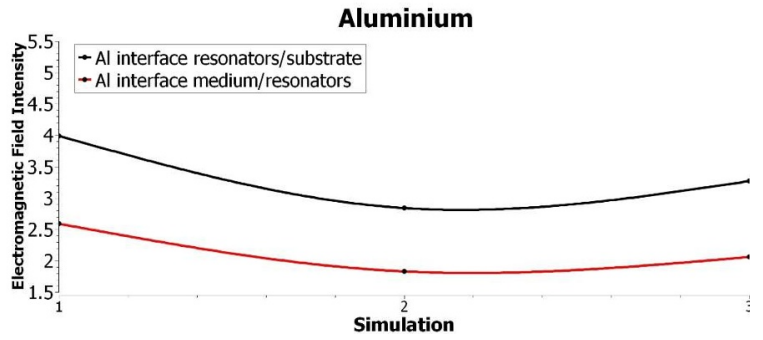

b)

Fig. 6. Electromagnetic field intensity on the two observation planes for U-shape resonators: a) silver $U$; b) aluminium.

Figure 7 presents the electromagnetic field at the two planes (at the interface medium/resonators and on the substrate around the resonator respectively) for metasurfaces with a 2D squared array of silver U-shape elements corresponding to simulation set 3 from table 1 .

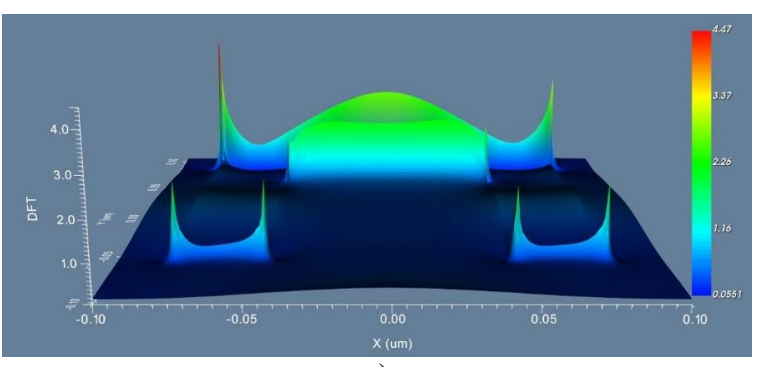

a)

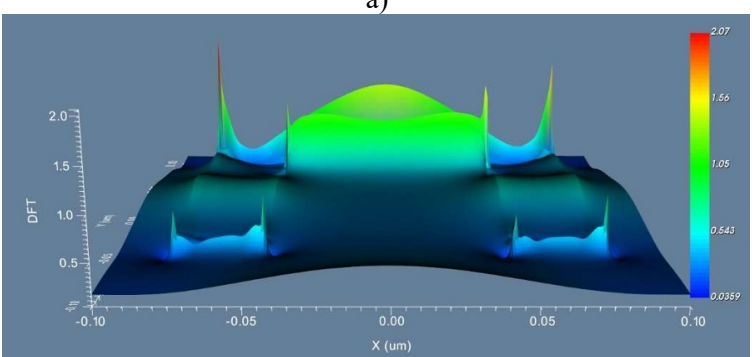

b)

Fig. 7. Electromagnetic field intensity for silver U-shape elements metasurface: a) interface medium/resonators; b) on the substrate around the resonator $-\mathrm{W}=10 \mathrm{~nm}, \mathrm{Wx}=110 \mathrm{~nm}$;

$$
\mathrm{Wy}=90 \mathrm{~nm} \text {. }
$$

Similar results were obtained for the emission wavelength of Rhodamine $6 \mathrm{G}-552 \mathrm{~nm}$. Here, the increase is around 4.48 times, meanwhile the increment at the medium/resonators interface is 2.25 times. Figure 8 present the simulation results at the medium/resonators interface.

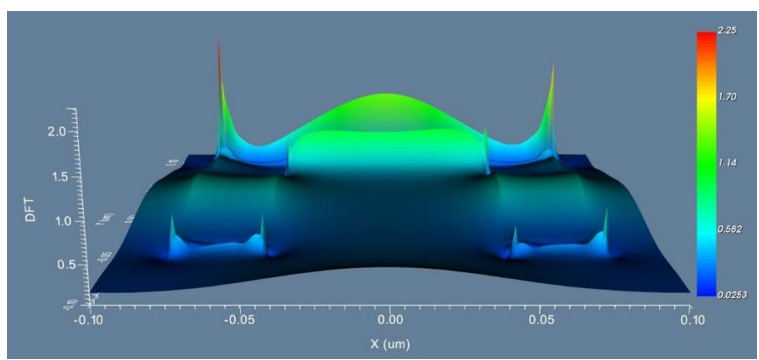

Fig. 8. Electromagnetic field intensity on the substrate around the resonator for silver U-shape elements metasurface with $\mathrm{W}=10 \mathrm{~nm}, \mathrm{Wx}=110 \mathrm{~nm} ; \mathrm{Wy}=90 \mathrm{~nm}$ parameters; at the Rhodamine $6 \mathrm{G}$ emission wavelength. 


\section{Conclusions}

In this paper we present an ample numerical analysis method to identify the optimum geometry, size and the best metal of the nano-antennas that compose a plasmonic metasurface, in order to obtain localized fluorescence enhancement. We investigated ten different types of metals, two shapes and various geometrical parameters for the nano-resonators composing the metasurface.

The best result for Rhodamine $6 \mathrm{G}$ excitation and emission, when the meta-atoms of the metasurface are nano-disks with $105 \mathrm{~nm}$ diameter in a rectangular array with a $110 \mathrm{~nm}$ period, are obtained using silver nanoresonators. The disadvantage of this geometry is the high aspect-ratio of the structures, thus making it almost impossible to fabricate.

In the case of U-shape nano-resonators, the best results are obtained with $90 \mathrm{~nm}$ base length, $110 \mathrm{~nm}$ arm length configured in a rectangular array with $20 \mathrm{~nm}$ period. This type of structure can be easily fabricated using electron beam lithography (EBL) and lift-off technique.

The promising local enhancement results and the available technology process make this type of structure suitable for biosensing application with possibility of binding the molecule labelled with fluorophores both on top of the nano-resonators and between them.

1. J. Hu, S. Bandyopadhyay, Y. Liu, L. Shao, Front. Phys. 8, 586087 (2021)

2. A. Vaskina, R. Kolkowski, A. Femius Koenderink, I. Staude, Nanophotonics 8 (7), 1151-1198 (2019)

3. D. Bouchet et.al, Phys. Rev. Applied 6, 064016 (2016)

4. R. H Siddique, S. Kumar, V. Narasimhan, H. Kwon, H. Choo, ACS Nano 13, 13775-13783 (2019)

5. R. Tomescu, C. Kusko, D. Cristea, R. Calinoiu, C. Parvulescu, SSELJ 2, 146-150, (2020)

Acknowledgment: This work was supported by a grant of the Romanian Ministry of Research, Innovation and Digitalization, CCCDI - UEFISCDI, project number PNIII-P2-2.1-PED-2019-1300, within PNCDI III 